\title{
Misdiagnosis of bilateral tubal pregnancy: a case report
}

\author{
Weifeng Li, Gang Wang ${ }^{*}$, Tiecheng Lin and Wenwen Sun
}

\begin{abstract}
Introduction: The incidence of bilateral tubal pregnancy is rising due to the increase of pelvic inflammatory disease and assisted reproductive techniques. Because the clinical manifestations of bilateral tubal pregnancy are not specific, we often ignore inspection of the other fallopian tube when focusing on the lesions, which may cause misdiagnosis.

Case presentation: A 33-year-old Chinese woman presented with vaginal bleeding after menopause and with an abnormality found by transvaginal ultrasound scan for which she underwent laparoscopy and salpingectomy. Unfortunately, she had to undergo a repetitive laparoscopic salpingotomy for the other tubal pregnancy due to misdiagnosis of her bilateral tubal pregnancy.

Conclusions: The incidence of unusual presentations of ectopic pregnancies has risen. Surgeons should always keep in mind the possibility of bilateral tubal pregnancy. An attentive examination of the pelvis, especially the two fallopian tubes, is necessary to avoid missing bilateral tubal pregnancy.
\end{abstract}

Keywords: Bilateral tubal pregnancy, Laparoscopy, Misdiagnosis

\section{Introduction}

Bilateral tubal pregnancy (BTP) is a very rare type of ectopic pregnancy. The incidence of bilateral tubal pregnancy is 1 in 725 to 1580 ectopic pregnancies and 1 out of 200,000 pregnancies [1]. The occurrence has tripled in the last decades with most cases being associated with previous assisted reproduction techniques (ART), with the use of intrauterine contraceptive devices (IUD), with pelvic inflammatory disease (PID), history of ectopic pregnancy or following tubal surgery $[2,3]$. We report a misdiagnosed case of BTP that occurred in The First People's Hospital of Foshan, China on 12 December 2013.

\section{Case presentation}

A 33-year-old Chinese woman presented to the gynecology clinic complaining of vaginal bleeding without abdominal pain. Her last menses was 42 days before the visit. She used no form of contraception. Our patient had no history of PID, no prior IUD, no use of fertility drugs and no pelvic surgery. Our patient had had conservative treatment in our

\footnotetext{
*Correspondence: Wallace1971@163.com

Department of Obstetrics and Gynecology, The First People's Hospital of

Foshan, No. 81, North Lingnan Street, Chancheng District, Foshan,

Guangdong 528000, China
}

hospital due to a left tubal pregnancy eight years before (a single-dose methotrexate (MTX) injection $(50 \mathrm{mg} / \mathrm{m} 2$ ) was administered, and she had an uneventful decrease in serum $\beta$-human chorionic gonadotropin (HCG) levels within two weeks) and one spontaneous abortion two years before. A physical examination revealed stable vital signs: blood pressure of $107 / 55 \mathrm{mmHg}$ and a pulse rate of $88 /$ min. Her abdomen was soft and nontender. A vaginal examination revealed bloody discharge and a nontender pelvis. Her serum level of $\beta$-HCG was $6993.1 \mathrm{IU} / \mathrm{L}$ and her progesterone level was $13.40 \mu \mathrm{g} / \mathrm{L}$. No gestational sac was detected in the uterine cavity by transvaginal ultrasound (TVUS) scan on 12 December 2013.

The presumptive diagnosis of ectopic pregnancy was made and our patient was admitted to our gynecology ward. Upon admission, her serum level of $\beta$-HCG increased to $13721.3 \mathrm{IU} / \mathrm{L}$. A $45 \mathrm{~mm} \times 29 \mathrm{~mm}$ right adnexal mass was found by TVUS examination (on 13 December 2013) (Figure 1), and an ectopic pregnancy was suggested. Nothing abnormal was detected in the left adnexal area. The endometrial thickness was $10 \mathrm{~mm}$ and the uterine cavity was empty. Having discussed the pros 


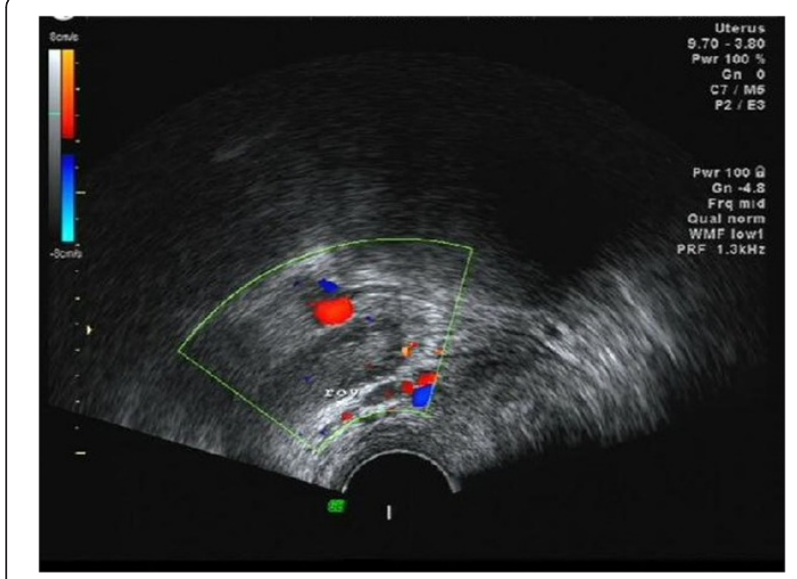

Figure 1 An ultrasound scan (on 13 December 2013) showed a right adnexal mass and nothing abnormal was detected in the left attachment area.

and cons of medical and surgical treatment options before the surgery, our patient and her family preferred the surgical treatment, and she insisted that she would like to preserve the integrity of the tube. After informed consent was obtained, a laparoscopy was performed, which revealed a $40 \times 50 \mathrm{~mm}$ nearly ruptured mass in the interstitial part of the right tube (Figure 2). A wedge resection of the right side of the uterine horn and partial (isthmus) resection of the right fallopian tube were done. An inspection of the left tube showed it was slightly thickened, which was considered to be a inflammatory sign because of the left tubal pregnancy eight years before. Pathology results confirmed placental villi in the right tube.

Her serum level of $\beta$-HCG was 7373.5IU/L on the first day after surgery and increased to 10522.3IU/L the next day. On 16 December 2013, an ultrasound scan showed a $24 \mathrm{~mm} \times 14 \mathrm{~mm}$ anechogenic image in her left fallopian tube, mild thickened endometrium, and a small amount of fluid collection in the Douglas Pouch (Figure 3). Our

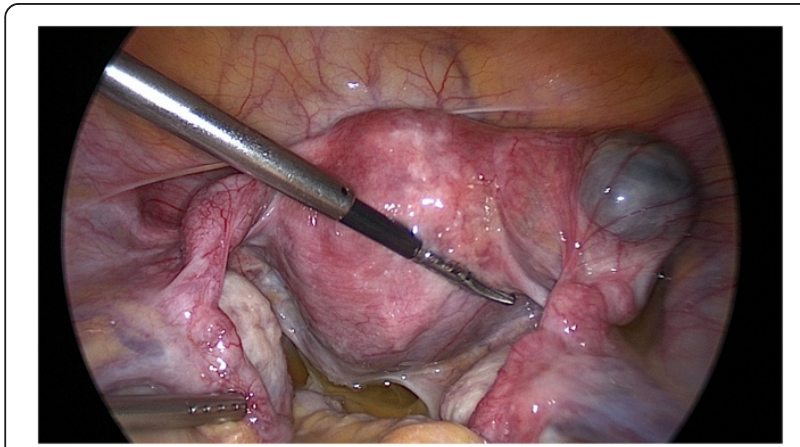

Figure 2 During the first surgery, a $40 \times 50 \mathrm{~mm}$ nearly ruptured right interstitial tubal pregnancy and a slightly thickened left tube were found.

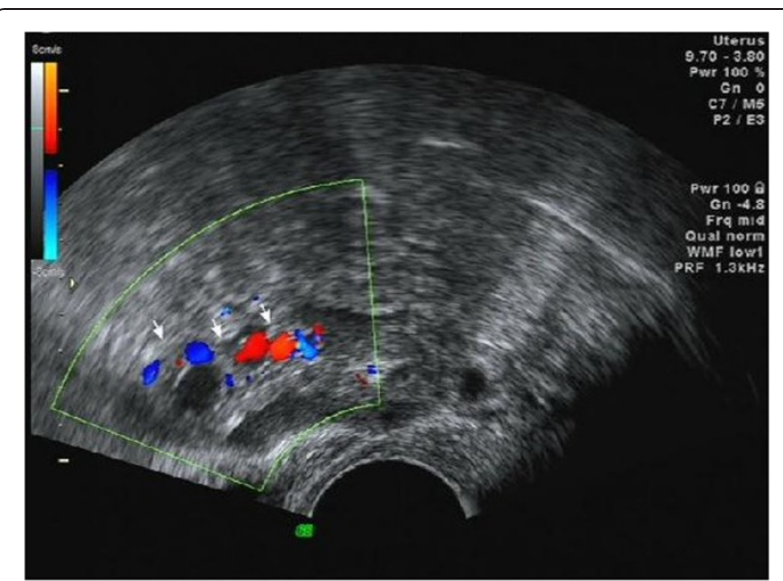

Figure 3 An ultrasound scan (on 16 December 2013) showed a $24 \mathrm{~mm} \times 14 \mathrm{~mm}$ left adnexal mass.

patient had no obvious discomfort after the surgery so that the expectant therapy was executed.

On 18 December 2013, her serum level of $\beta$-HCG rose to $13721.3 \mathrm{IU} / \mathrm{L}$ and her progesterone level was $8.94 \mu \mathrm{g} / \mathrm{L}$. A $26 \mathrm{~mm} \times 16 \mathrm{~mm}$ left adnexal mass and a small amount of fluid was detected on TVUS examination. The endometrial thickness was $11 \mathrm{~mm}$ and the uterine cavity was empty (Figure 4). Our patient was considered for BTP. A laparoscopy was performed after informed consent was obtained. A $30 \times 20 \mathrm{~mm}$ unruptured left ampullary ectopic pregnancy was found (Figure 5). A left linear salpingostomy and curettage were performed. Pathology results confirmed placental villi in the mass of the left tube and that there were no placental villi in the curettage specimen. Our patient was discharged home two days after surgery, in good condition. Her $\beta$ HCG serum level was $4128.0 I U / L$ when she left

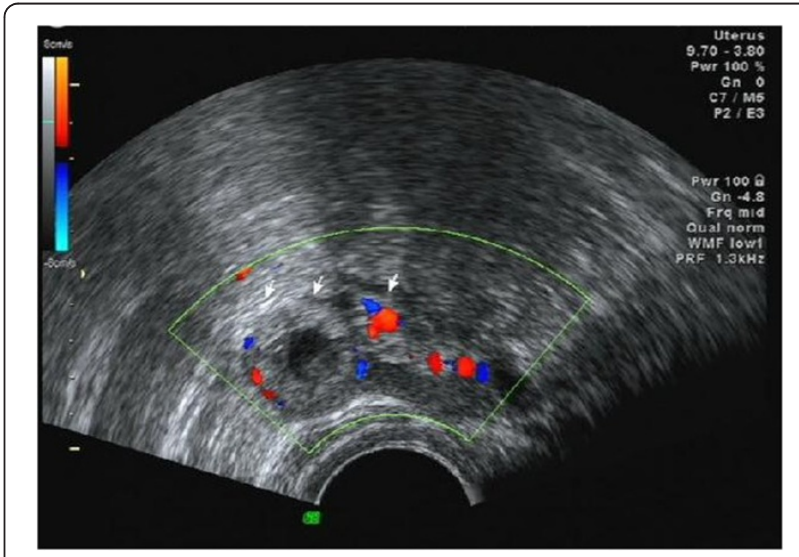

Figure 4 An ultrasound scan (on 18 December 2013) showed a $26 \mathrm{~mm} \times 16 \mathrm{~mm}$ left adnexal mass. 


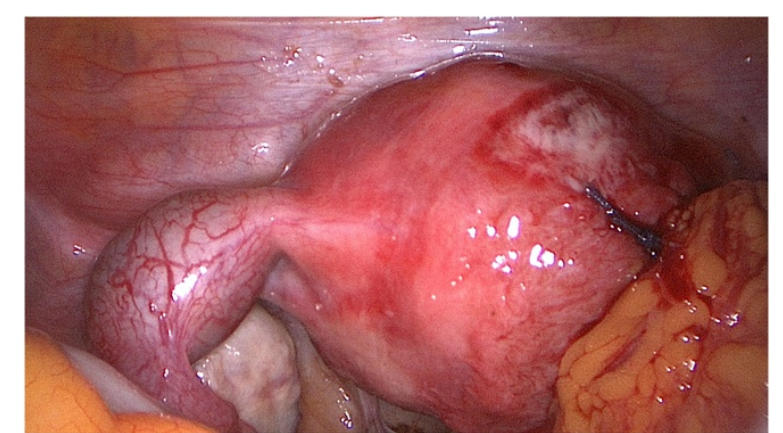

Figure 5 During the secondary surgery, a $30 \times 20 \mathrm{~mm}$ unruptured left ampullary pregnancy was found.

hospital. She was followed up until her $\beta$-HCG serum level was normal.

\section{Discussion}

An increase in the incidence of PID and increase in ARTs has caused a rise in BTP. BTP is the rarest form of ectopic pregnancy [4]. The incidence is reported to be five in one million deliveries [5]. Many cases are as a result of ARTs [6-9]. Nevertheless, it can be seen in normal natural menstrual cycles.

The mechanisms of BTP may include multiple ovulation, transperitoneal migration of trophoblastic tissue from one tube to another, or superfetation [10].

It is hard to diagnose BTP. The diagnosis is rarely confirmed before surgery. The clinical presentation of BTP is nonspecific. There are no unique clinical features to differentiate it from unilateral ectopic pregnancy. The diagnosis of BTP is hard to make based on serum levels of $\beta$-HCG, progesterone level and transvaginal ultrasound scan, particularly for nonsimultaneous bilateral tubal pregnancy. Sometimes, preoperative diagnosis made by imaging would cause misdiagnosis. To take our case as an example, the left ectopic pregnancy was neglected once the right pregnancy had been confirmed, which lead to our patient undergoing a secondary surgery. Most BTPs are diagnosed in the operating room. During the operation, once a gestational sac is found, a second gestational sac is not expected, and elevated serum levels of $\beta$-HCG are easily ignored. A lack of careful examination of the contralateral tube leads to misdiagnosis, just like in our case. Surgeons should always keep in mind the possibility of BTP, especially when facing a patient who has risk factors like PID, history of ectopic pregnancy and ART. Direct inspection of the contralateral tube in the operating room is the most effective method of diagnosing the second ectopic pregnancy. Laparoscopy is the gold standard for diagnosis of ectopic pregnancy, including BTP. The criteria for diagnosis of BTP were first suggested by Fishback [11] and later revised by Norris [12] who stated that microscopic demonstration of chorionic villi in each tube was sufficient for the diagnosis. A histopathological examination is essential to confirm the diagnosis, with the identification, at least, of chorionic villi in both tubes.

With regard to treatment for BTP, no guidelines are presently available on this topic. Laparoscopy is a diagnostic management and also a therapeutic process. The most proper and safest way to deal with BTP may be laparoscopic salpingostomy. But if both tubes are badly damaged or actively bleeding, bilateral salpingectomy may be a more suitable option [13]. For those infertile patients who received ART and were diagnosed with BTP, removal of both fallopian tubes might be a solution to exclude potential nonsimultaneous bilateral tubal pregnancy. Otherwise, careful examination of the contralateral tube during surgery and close follow-up of the $\beta$-HCG serum level after surgery facilitates discovery of potential nonsimultaneous bilateral tubal pregnancy for those patients who desire to preserve the other tube.

\section{Conclusions}

This is a rarely seen case of spontaneous BTP. The incidence of unusual presentations of ectopic pregnancies has risen along with an increase in assisted reproductive techniques and pelvic inflammatory disease. A cautious examination of both fallopian tubes is necessary to avoid missing bilateral tubal pregnancy, especially for the patient who has risk factors like PID, history of ectopic pregnancy and ART. Making the diagnosis of BTP before or during surgery is critical so that the patient can avoid a secondary surgery. How to diagnose and treat BTP properly is a big responsibility for physicians. The purpose of this manuscript is to promote research into the epidemiology of BTP, improve diagnosis, and find the appropriate treatment.

\section{Consent}

Written informed consent was obtained from the patient for publication of this case report and any accompanying images. A copy of the written consent is available for review by the Editor-in-Chief of this journal.

\section{Competing interests}

The authors declare that they have no competing interests.

\section{Authors' contributions}

WL collected information and wrote the manuscript. GW analyzed and interpreted the patient data regarding diagnosis. TL and WS performed the surgery. All authors read and approved the final manuscript.

Received: 15 February 2014 Accepted: 13 August 2014

Published: 14 October 2014 
References

1. Al-Quraan GA, Al-Taani MI, Nusair BM, Arafat MR, Khateeb MM: Spontaneous ruptured and intact bilateral tubal ectopic pregnancy. East Mediterr Health J 2007, 13:972-974.

2. Bettocchi S, Nappi L, Ceci O, Vimercati A, Selvaggi L, Cormio G, Vicino M: Simultaneous bilateral tubal pregnancies and intrauterine pregnancy with five fetuses. J Am Assoc Gynecol Laparosc 2004, 11:195-196.

3. Shenoy JV, Choudhary V, Giles RW: Bilateral ectopic pregnancy. J Obstet Gynaecol 2005, 25:612-613.

4. Andrews J, Farell S: Spontaneous bilateral tubal pregnancies: a case report. J Obstet Gynecol Can 2008, 30:51-54.

5. Edelstein MC, Morgan MA: Bilateral simultaneous tubal pregnancy: case report and review of literature. Obstet Gynecol Surv 1989, 44:250-252.

6. Musarrat J, Babar A, Jakimiuk J: Bilateral ectopic pregnancy following ovulation induction. JPM 2010, 24:160-162

7. Tadeusz I, Wojciech G, Attur J: Bilateral ectopic tubal pregnancy, following in vitro fertilization (IVF). Folia Histochem Cytobiol 2009, 47:147-148.

8. Burgos-San Cristobal DJ, Agirregoikoa JA, Albisu M: Simultaneous bilateral ectopic pregnancy after IUI. Rev Iberoam Fertile Reprod Hum 2004, 21:349-353.

9. Ghaffari F, Eftekhari Yazdi P, Kiani K: A case report of bilateral tubal ectopic pregnancy following day 5 embryo transfer. Arch Med Sci 2011, 7:1087-1088.

10. Tabachnikoff RM, Dada MO, Woods RJ, Rohere D, Myers CP: Bilateral tubal pregnancy: a report of an unusual case. J Reprod Med 1998, 43:707-709.

11. Fishback HR: Bilateral simultaneous tubal pregnancy. Am J Obstet Gynecol 1939, 37:1035-1037.

12. Pezzani M: A case of simultaneous interstitial bilateral pregnancy. Acta Gent Med Gemellol 1976, 35:325-327.

13. Ataíde Lobo R, Patrício L, Milheiras E, Cordeiro A, Hermida M: Bilateral tubal Ectopic PregancyGravidez ectópica t ubária bilateral. Acta Obstet Ginecol Port 2012, 6:141-144.

doi:10.1186/1752-1947-8-342

Cite this article as: Li et al:: Misdiagnosis of bilateral tubal pregnancy: a case report. Journal of Medical Case Reports 2014 8:342.

\section{Submit your next manuscript to BioMed Central and take full advantage of:}

- Convenient online submission

- Thorough peer review

- No space constraints or color figure charges

- Immediate publication on acceptance

- Inclusion in PubMed, CAS, Scopus and Google Scholar

- Research which is freely available for redistribution 